\title{
Speeding Up a Genetic Algorithm for EPR-Based Spin Label Characterization of Biosystem Complexity
}

\author{
Aleh A. Kavalenka, ${ }^{\dagger}$ Bogdan Filipič, ${ }^{\ddagger}$ Marcus A. Hemminga, ${ }^{\S}$ and Janez Štrancar*,॥ \\ Department of Systems Analysis, Belarusian State University, F. Skorina Avenue 4, Minsk 220050, Belarus, \\ Department of Intelligent Systems and Laboratory of Biophysics, Solid State Physics Department, Jožef \\ Stefan Institute, Jamova 39, SI-1000 Ljubljana, Slovenia, and Laboratory of Biophysics, Wageningen \\ University, Dreijenlaan 3, NL-6703 HA Wageningen, The Netherlands
}

Received April 28, 2005

\begin{abstract}
Complexity of biological systems is one of the toughest problems for any experimental technique. Complex biochemical composition and a variety of biophysical interactions governing the evolution of a state of a biological system imply that the experimental response of the system would be superimposed of many different responses. To obtain a reliable characterization of such a system based on spin-label Electron Paramagnetic Resonance (EPR) spectroscopy, multiple Hybrid Evolutionary Optimization (HEO) combined with spectral simulation can be applied. Implemented as the GHOST algorithm this approach is capable of handling the huge solution space and provides an insight into the "quasicontinuous" distribution of parameters that describe the biophysical properties of an experimental system. However, the analysis procedure requires several hundreds of runs of the evolutionary optimization routine making this algorithm extremely computationally demanding. As only the best parameter sets from each run are assumed to contribute into the final solution, this algorithm appears far from being optimized. The goal of this study is to modify the optimization routine in a way that 20-40 runs would be enough to obtain qualitatively the same characterization. However, to keep the solution diversity throughout the HEO run, fitness sharing and newly developed shaking mechanisms are applied and tested on various test EPR spectra. In addition, other evolutionary optimization parameters such as population size and probability of genetic operators were also varied to tune the algorithm. According to the testing examples a speed-up factor of 5-7 was achieved.
\end{abstract}

\section{INTRODUCTION}

Complexity is one of the basic properties of natural biological systems. It qualitatively describes the number of (biochemical or biophysical) patterns/solutions that coexist in a system. In a pure system, only one solution can describe the entire system, whereas in complex systems distributions of solutions can exist (see Figure 1). The complexity of a biological membrane, for example, originates in its biochemical composition of a few hundred lipids and many different proteins - channels and pumps, as well as membrane enzymes and receptors. In such a system, the constituents exhibit different interactions to each other, from local steric and van der Waals to more long-ranged Coulomb and dipolar interactions. The intensity and orientation of these interactions strongly depend on the type of interacting molecules as well as the potentials of the neighboring molecules. All these parameters make the biological membrane a very complex system in which many motional patterns can be found.

EPR spectroscopy in combination with nitroxide spin labeling (SL-EPR) has proven to be a powerful technique

* Corresponding author phone: +386 147732 26; fax: +3861477 31 91; e-mail: Janez.strancar@ijs.si.

Belarusian State University.

$\doteqdot$ Department of Intelligent Systems, Jožef Stefan Institute.

$\S$ Wageningen University.

"Laboratory of Biophysics, Solid State Physics Department, Jožef Stefan Institute.

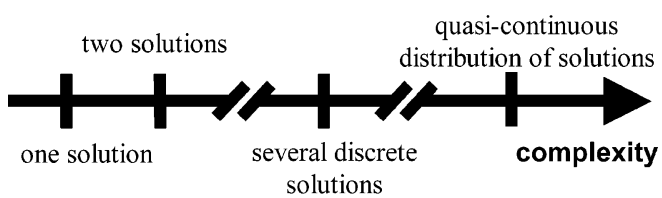

Figure 1. Biosystem complexity axis of increasing complexity from simple single-solution to quasicontinuous distribution of solutions.

for the exploration of heterogeneity and motion in biological systems. ${ }^{1,2}$ The time scale of SL-EPR appears to be in the nanoseconds range, which is exactly the range needed to observe possible motional anisotropy of local rotational motions through motional averaging. The difference in anisotropy of rotational motion can be used to distinguish lateral domains together with other spectroscopic parameters such as the rate of motion, polarity, spin-spin broadening, etc. However, to determine the picture of the actual heterogeneity within biomembranes, a special methodology that includes advanced spectral analysis and inverse-problem solving techniques needs to be applied. ${ }^{3}$ Such an analysis is based on mathematical modeling, spectrum fitting, and spectral parameter optimization by means of evolutionary computation. A large amount of information evolves from such an approach. Therefore a special method of solution condensation called GHOST was developed. ${ }^{1}$ It incorporates solution density filtering, $\chi^{2}$ goodness filtering, solution-space slicing, and domain determination, leading to a graphical presentation of the system parameters. This advanced ap- 


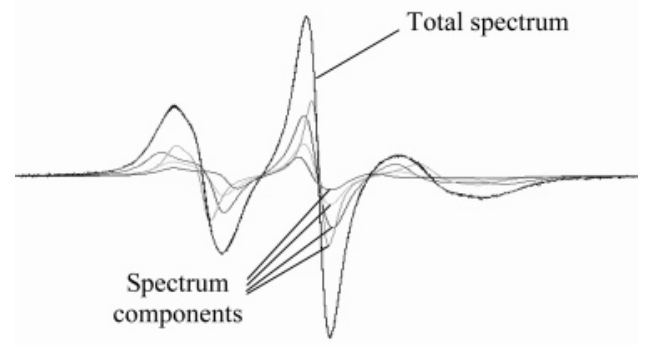

Figure 2. Superimposed four component EPR spectrum. The total EPR spectrum is a sum of four spectral components derived within a described simulation model and determined by four sets of the following spectral parameters $\left\{\vartheta, \varphi, \tau_{c}, W, p_{\mathrm{A}}\right.$, Prot, $\left.d\right\}$.

proach named Hybrid Evolutionary Optimization (HEO) was shown to be powerful enough to study complex heterogeneous systems, ${ }^{1}$ but the computational demand appeared to be an obstacle for wider usage of the method.

The core of the problem lies in the optimization routine. To obtain a reliable result even in the case of quasicontinuous problems, the HEO procedure has to be executed at least 200 times. Each particular run consists of 100 generations with a population size of 300 candidate solutions that are exposed to various genetic operators. Since an average operator spends up to 10 spectrum calculations, HEO on average spends 60 million spectrum calculations. As a single spectrum calculation takes around $10 \mathrm{~ms}$ on a 1 GFLOPS processor, this results in $200 \mathrm{~h}$ of computer time spent for a single characterization. Therefore, our aim was to enhance the HEO routine to speed up the approach to make it more applicable.

\section{THEORY AND METHODOLOGY}

EPR Spin Labeling. EPR spectroscopy in combination with spin labeling can be applied to study the properties of biological membranes in a nondestructive way. In this approach spin-labeled analogues of different molecules are introduced into a system to report about their structural and motional properties. Since the nitroxide moiety is a small perturbation to the whole molecule, one can approximate that the description derived from spin probes is a reasonable approximation for the nonlabeled molecules. This fact enables us to use EPR to explore biological systems in vivo so that there is no need for (bio)chemical extraction of the subsystem of interest. In this way, various coexisting states of the system can be detected and characterized.

As was mentioned in the Introduction, EPR spin labeling inherits a unique sensitivity to the motional and polarity properties of the labeled molecules providing an opportunity to extract information on structure and dynamics of the lipids and membrane proteins (i.e. restriction and rate of rotational motions, relative membrane locations, and oxygen profile). The complexity of such a system results in a large number of solutions superimposed in the EPR spectrum of such a labeled system (Figure 2).

EPR Spectrum Modeling. Generally, to describe the EPR spectra of spin labels, the stochastic Liouville equation should be used. ${ }^{4-6}$ However, under physiological conditions the majority of the local rotational motions is fast with respect to the EPR time scale-as calculated by numerous molecular dynamics simulations - and therefore the fast motional ap- proximation can be applied, reducing the computational demand by a factor of 100 .

Since the basic approach has been already discussed elsewhere, ${ }^{7,8}$ we will emphasize only the physical background of the spectral parameters involved in our calculations. First, one or two parameters are used in partial averaging of the rotational motion. While averaging the magnetic properties of the spin Hamiltonian for spin probes directed at every allowed direction with respect to the external magnetic field, an order parameter $S$ or opening cone angle $\vartheta$ (that defines the maximal tilt angle) and asymmetry cone angle $\varphi$ (that describes the maximal restriction of spinning) will be applied. Second, the traces of the interaction tensors $\mathbf{g}$ and $\mathbf{A}$ are linearly corrected with $p_{\mathrm{A}}{ }^{9}$ and Prot parameters to take into account the effects of polarity and proticity, respectively. Third, when calculating the convolution of the magnetic field distribution and basic line shape, in addition two line width parameters are applied: a Lorentzian-type line is defined in the motional narrowing approximation ${ }^{10}$ with a single (effective) rotational correlation time, $\tau_{c}$, and an additional broadening constant $W$. The latter arises primarily from unresolved hydrogen superhyperfine interactions and contributions of paramagnetic impurities (e.g. oxygen), external magnetic field inhomogeneities, field modulation effects, and spin-spin interaction.

To take into account the superposition of motional/polarity patterns, this basic set of six line shape parameters $\vartheta, \varphi, \tau_{c}$, $W, p_{\mathrm{A}}$, and Prot is expanded for the number of spectral components $N_{\mathrm{c}}$. In addition there are $N_{\mathrm{c}}-1$ weights $d$ of these spectral components. Altogether, there are $7 N_{\mathrm{c}}-1$ spectral parameters, which have to be tuned by the optimization routine. Taking into account the resolution limit of SL-EPR which is around 30 parameters, this allows the usage of at most 4 spectral components.

Optimization. An optimization routine is used to find the set of spectral parameters that produces the best fit to the experimental spectrum. The goodness of fit (optimization objective function) was chosen to be the reduced $\chi^{2}$ criteria

$$
\chi^{2}=\frac{1}{N-p_{i=1}} \sum_{\frac{\left(y_{i}^{\exp }-y_{i}^{\text {sim }}\right)^{2}}{\sigma^{2}}}
$$

where $y^{\exp }$ and $y^{\text {sim }}$ are the experimental and simulated data, respectively, $\sigma$ is the standard deviation of the experimental points, $N$ is the number of spectral points, and $p$ the number of model parameters.

For the optimization, HEO routine, a combination of the Genetic Algorithm (GA) with Downhill-Simplex local search was applied. Since the optimization scheme is presented elsewhere, ${ }^{11}$ we only briefly report on the implemented algorithm. The routine starts with a random initialization of solutions and continues with the tournament selection and application of genetic operators for 100 generations. The 3 -point crossover with probability of 0.7 and uniform mutation with probability of 0.01 are applied together with certain knowledge-based operators and local improvements (performed with Downhill-Simplex with probability of 0.002 , see Figure 3). ${ }^{1,11}$ The elite set ( $2 \%$ of the population size) is used to keep track of the best individuals found so far. One HEO consists of 100 generations with a population size of 300 individuals and provides the best parameter set found. 


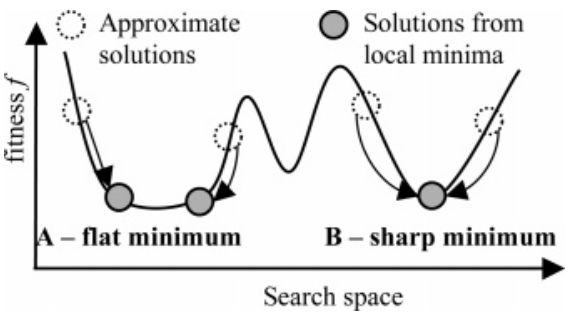

Figure 3. Schematic presentation of parameters search space and the effect of the local mutation procedure responsible for finetuning. Due to the noisy spectra and finite resolution of the local optimization routine starting approximations (white circles) are optimized into more accurate solutions (gray circles) according to the local phase-space landscape. (a) In case of a flat valley (plateau in multidimensional space), the results of the local optimization routine strongly depend on the starting approximation. (b) In case of sharply defined minimum, local optimization routine provides similar results independently of starting approximation unless starting approximation is too far from the local minimum.

In the $200 \mathrm{HEO}$ runs a group of best parameter sets can be accumulated. This information is then filtered, grouped, and graphically presented with a so-called GHOST condensation algorithm.

Taking only one best parameter set from each run can be a waste of computer time. In fact, HEO converges to the best solution region within 20-80 generations, thus creating a great number of similar solutions after 100 generations. Therefore, HEO was modified to increase the solution diversity within the population while preserving the same level of convergence rate. In such a case, it should be possible to include more than one parameter set into the final group of solutions and consequently rely on a smaller number of runs.

To maintain the population diversity throughout the GA generations and not to affect convergence, one should modify the selection scheme or add new operator(s) to keep the diversity within the population. To do that, one should clearly understand the HEO as well as the problem search space.

Parameters Search Space. The optimization process should be thought of as searching for the minima in the landscape of the parameter search space (phase-space), which may contain both local and global minima. A powerful optimization routine should be able to find global minimum(a), which can be of different types (Figure 3), i.e., welldefined minima (Figure $3 b$ ) or a flat minimum valley minima (Figure 3a). An optimization routine should therefore keep convergence to the minima of type $\mathbf{B}$ (discrete problems) and maintain the diversity to be able to reveal the minimum valleys (in continuous problems) already in a single run.

Population Diversity in GA. Genetic algorithms are general purpose global search algorithms that use principles of natural genetics. Simultaneously, a population of possible solutions is being optimized. A simple genetic algorithm (SGA) ${ }^{12}$ is suitable for finding the optimum of a unimodal function in a bounded search space. However, both analysis and experiments show that the SGA cannot find multiple global maxima of a multimodal function ${ }^{12-14}$ or a function with a flat global minimum, which is an extreme limit of the multimodal function. This limitation can be overcome by a mechanism that creates and maintains several subpopulations within the search space, referred to as "niching methods". There exist sequential niching methods; ${ }^{15,16}$ parallel niching methods (sharing, ${ }^{17}$ crowding, ${ }^{14,18}$ and clearing ${ }^{13}$ );

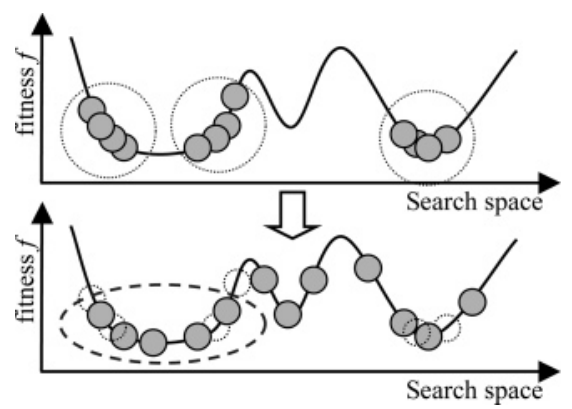

Figure 4. Schematic presentation of the fitness sharing operator function. Top: In a nonsharing routine crowding at the local minima is allowed, since there is no operator that would maintain diversity. Bottom: In a sharing-routine, fitness function is increased according to the density of solution, aiming to prevent crowding.

speciation methods ${ }^{19-21}$ and clustering $;^{22,23}$ and multipopulation methods ${ }^{24}$ (island models ${ }^{25,26}$ and migration models ${ }^{27}$ ).

Another way to find multiple optima is to make several runs of an ordinary GA. In each run the GA typically converges to a different optimum. Thus, several optima are found. ${ }^{28}$ Exactly this strategy was used in the previous multiple HEO-based approach.

Since the methods that assume creating subpopulations do not match with our specific problem, we chose the sharing parallel niching method to maintain diversity within a single run together with a multiple run approach.

Sharing. Sharing ${ }^{14,17}$ requires that fitness is shared as a single resource among similar individuals in a population of solutions. ${ }^{29}$ The fitness sharing method modifies the search landscape by changing the fitness function (2), i.e. the value of $\chi^{2}$, in densely populated regions ${ }^{30}$

$$
f^{\prime}(j)=\frac{f(j)}{\sum_{i=1}^{n} \xi(d[i, j])}
$$

where the sharing function $\xi$ is a function of distance $d[i, j]$ between two population elements and can be defined as

$$
\xi(j)= \begin{cases}1-\left(\frac{x}{\sigma_{\text {share }}}\right)^{\alpha} ; & x<\sigma_{\text {share }} \\ 0 ; & \text { otherwise }\end{cases}
$$

It returns ' 1 ' if the elements are identical and ' 0 ' if they cross some threshold of dissimilarity, specified by constant $\sigma_{\text {share. }}$. Here $\alpha$ is a constant, which regulates the shape of the sharing function. As a result of the sharing operator application, the population becomes better distributed in the search space which improves the population diversity (Figure 4).

Shaking. Shaking is a new operator that was developed to provide small Gaussian-like deviations to the spectral parameters (Figure 5) before the crossover operator is applied. The shaking algorithm prevents "grid" formation and preserves the diversity in the solution population (for explanation of the grid problem see Discussion section).

Projection Principle and GHOST Condensation. The large amount of solutions resulting from the multiple HEO runs should be condensed and grouped together to construct a discrete or quasicontinuous description of the system. If the proposed model complexity (4 spectral components in our case) is sufficient to describe the system, the final description is also discrete. However, when the proposed complexity is lower than in reality, the model tries to describe 

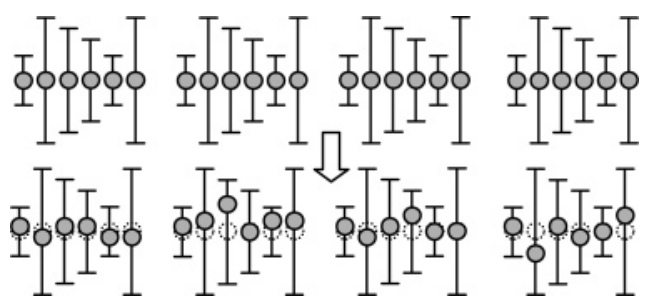

Figure 5. Schematic presentation of the Gaussian shaking operator. Shaking operator implies a Gaussian random generator that provides a small deviation to the value of each parameter. The error bars indicate the width of Gaussian probability distribution of these deviations. The standard relative uncertainties of the spectral parameters $\left\{\vartheta, \varphi, \tau_{c}, W, p_{\mathrm{A}}\right.$, Prot, $\left.d\right\}$ are $\{0.02,0.02,0.04,0.035$, $0.035,0.04,0.02\}$, respectively, which follow average uncertainties that are found empirically for these parameters within the simulation model.
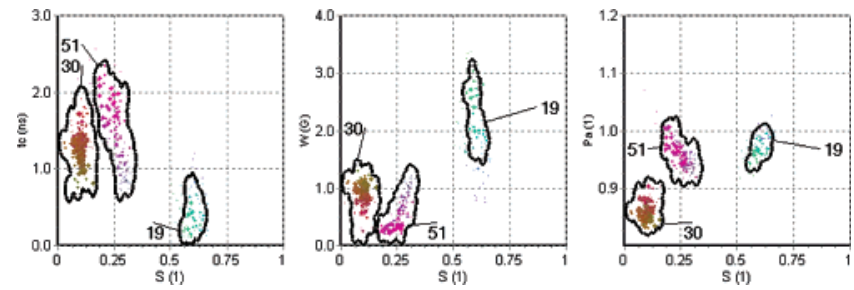

Figure 6. An example of the GHOST solution presentation of the spin labeled horse neutrophils taken from bronchoalveolar fluid (BAL) from horses suffering from the chronic obstructive pulmonary disease (COPD). Horses were sedated with medetomidine purchased from Domosedan (Turku, Finland). A 2.5-m long endoscope was introduced through the precleaned and topically anesthetized nostril and advanced until it wedged in a bronchus. Three hundred milliliters of prewarmed sterile physiological saline solution was infused through the biopsy channel into the bronchus and immediately reaspirated into a sterile flask cooled in ice. Polymorphonuclear leukocytes were isolated from whole BAL samples, spin labeled with MeFASL(10,3), centrifuged, transferred to quartz capillary, and measured at Bruker ELEXSYS E500 9.6 $\mathrm{GHz}$ spectrometer (field sweep of $10 \mathrm{mT}$; modulation: $0.15 \mathrm{mT}$, $100 \mathrm{kHz} ; 5$ scans of $40 \mathrm{~s}$ with $40 \mathrm{~ms}$ of time constant), fitted with EPRSIM BBW software and characterized using GHOST condensation procedure.

the most important features of the system (EPR spectra in our work). In this case, the landscape at the point of the global minimum changes into a flat valley, and consequently, HEO needs to resolve the distribution of solutions describing this optimum region of the parameter search space. In this way, multiple-HEO approach incorporates the "projection principle" idea. ${ }^{1,3}$

After solution filtering according to the local solution density and goodness of fit, performed in the same way as defined before, ${ }^{1}$ the GHOST condensed results are presented in $2 \mathrm{D}$ cross-sections $\left\{S-\tau_{c}, S-W, S-p_{\mathrm{A}}\right\}$ (Figure 6). The color of any solution point in the GHOST diagram is defined by RGB specification, where the intensity of each color component (red, green, blue) represents the relative value of the spectral parameters $\tau_{c}, W$, and $p_{\mathrm{A}}$ in their definition intervals $\{0-3 \mathrm{~ns}\},\{0-4 \mathrm{G}\}$, and $\{0.8-1.2\}$, respectively (Figure 7). This technique enhances the possibility to distinguish groups of solutions and to explore optimized values of model parameters.

The most important property of the GHOST algorithm is that there is no need to define the complexity (the number of different motional patterns) in advance-it comes out automatically from the GHOST condensation and graphical presentation.

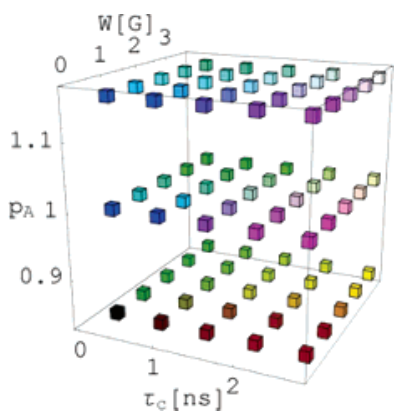

Figure 7. Color legend. The RGB (red, green, and blue) color of any particular solution point codes the relative values of parameters $\tau_{c}, W$, and $p_{\mathrm{A}}$ in their definition intervals.

Table 1. Optimization Parameters after 200 and 20 Runs for the Real Membrane Spectrum ${ }^{a}$

\begin{tabular}{ccc}
\hline criteria & 200 runs & 20 runs \\
\hline$\chi^{2}{ }_{\min }$ & 3.4 & 4.1 \\
$\sigma\left(\chi^{2}\right)$ & 2.0 & 1.9 \\
$\rho_{\max }$ & 64.2 & 71.5
\end{tabular}

${ }^{a}$ For the experimental preparation see the caption to Figure 6.

\section{RESULTS AND DISCUSSIONS}

Evaluation Criteria. To judge the success of the modification of the HEO algorithm the following criteria were selected: GHOST quality (solution diversity, solution domains determination, model parameters distribution); minimal fitness achieved in $\chi_{\text {min }}^{2}$, and fitness deviation $\sigma\left(\chi^{2}\right)$, that is $40 \%$ of the best $\chi^{2}{ }_{\min }$ values; runs contribution histograms; and maximal detected solution density $\rho_{\max }$. To check the generality of the new algorithm we analyzed two types of EPR spectra: experimental ones (from membranes and membrane proteins) and synthetic (discrete and continuous).

Multiple Runs. Before making any implementation changes in the code, we simply reduced the number of HEO runs from 200 to 20 and increased the contribution of each run (more than one best parameter set). The results for a typical experimental spectrum are shown in Figure 8 where the GHOST diagram (Figure 8b) and contribution histogram (Figure 8c) are compared with the original GHOST diagram based on the 200 runs (Figure 8 a). It can be easily seen that this is not the right way to reduce the computational demand of the problem. With the modified approach, the GHOST diagram (Figure 8b) does not resemble the original one (Figure 8a). In addition it can be seen that only a few runs (such as the first, seventh, ninth, and seventeenth) contribute to the GHOST presentation as it is shown by runs contribution histogram in Figure 8c, whereas the other runs (i.e. the third, fourth, tenth, etc.) have no contribution at all. This causes the loss of solution diversity, a worse distribution of $\chi^{2}$ (see minimum value and distribution width in "20 runs" column of Table 1), and a wrong solution domains determination (Figure 8b). In addition one also can see a higher solution density as a consequence of the crowding in the search space. An even worse result is achieved when the modified "20 runs" approach is tested on a continuous problem: compare original "200 runs" (a) and "20 runs" (b) in Figure 11. It can be easily seen that the results do not meet the original GHOST distribution. The bad GHOST picture arises from the fact that the contribution of the runs is extremely uneven (Figure 12b), originating in a solution crowding. 
(a)

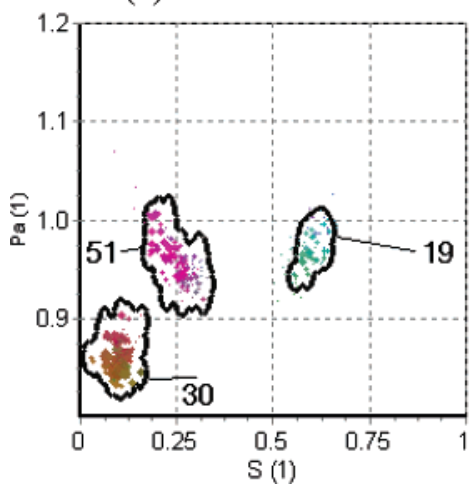

(b)

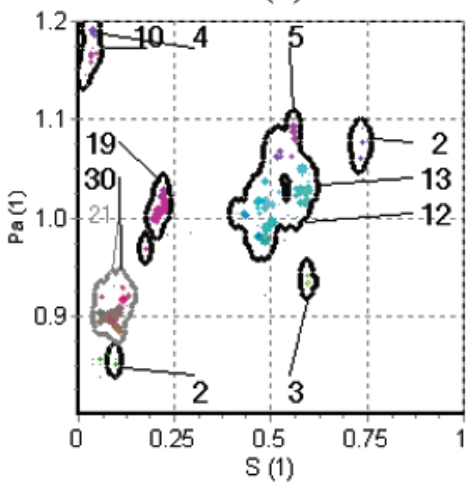

(c)

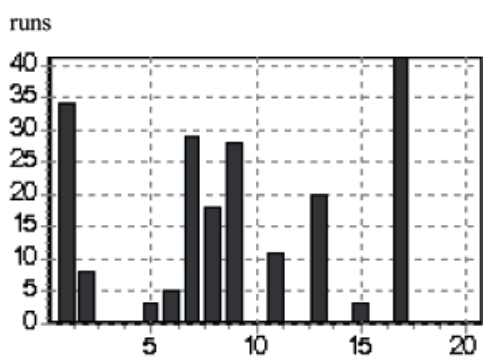

Figure 8. Typical characterization of spin labeled real membrane (see the caption to Figure 6): (a) GHOST as a result of 200 runs of HEO where only one solution is extracted from a single run; (b) GHOST as a result of 20 runs of the same HEO algorithm where on average 10 solutions are taken from each run; (c) runs contribution histogram for the case of 20 runs where the number of runs is shown along the $x$-axis and number of solution (taken from a particular run) along the $y$-axis.

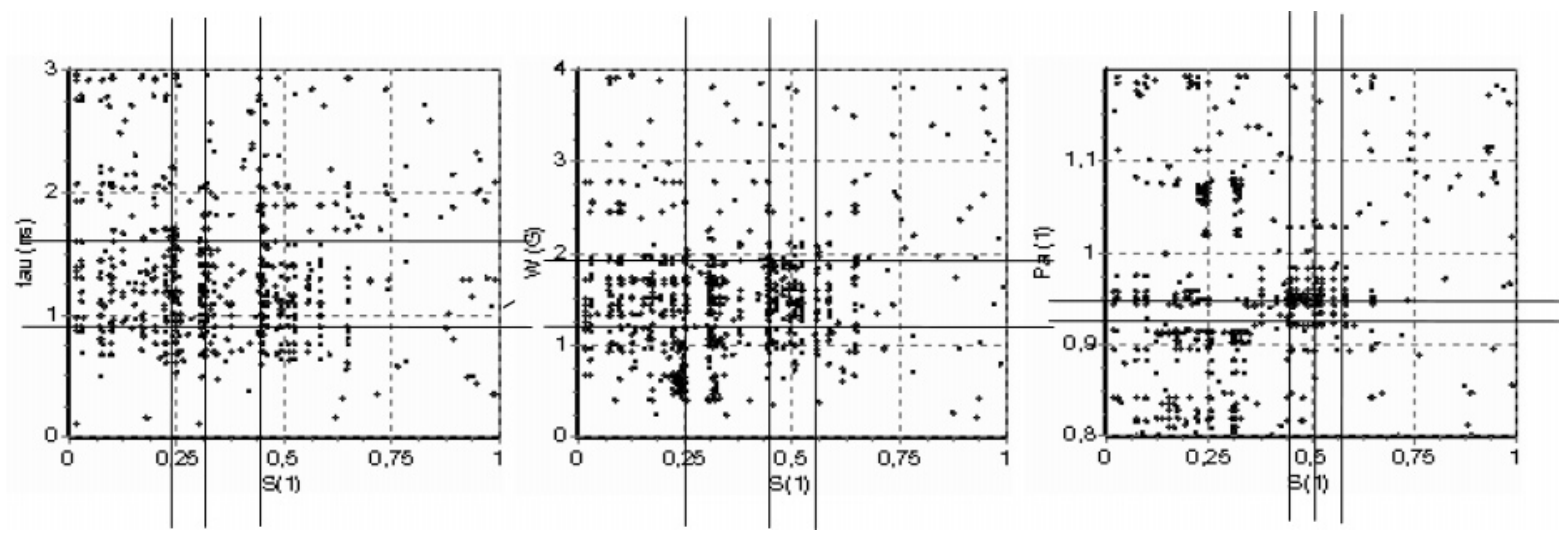

Figure 9. Schematic presentation of the "grid" problem for three cross-sections of the phase-space. Due to the standard multipoint crossover, subgroups of parameters are "transferred" between generations untouched, resulting in a gridlike distribution of the GHOST solution (single run). The lines indicate very high vertical and horizontal densities of solutions that evolve from copying of parts of parameter sets within the optimization routine.

(a)

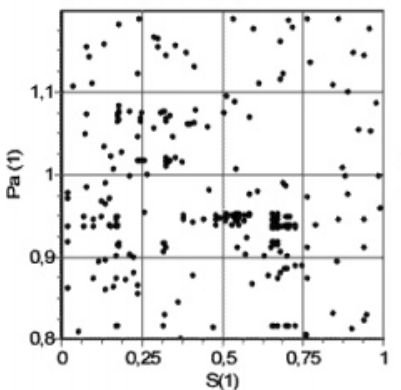

(b)

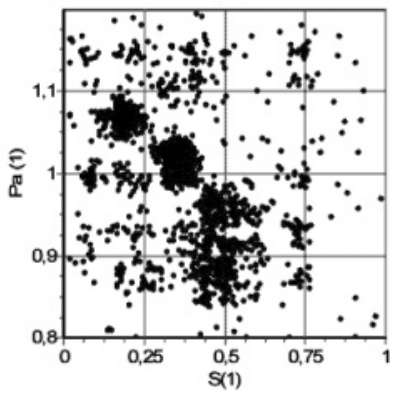

Figure 10. Single run GHOSTs (with population size 600): (a) original version with crowding problem-several solutions are crowded in many regions and (b) version with shaking that maintains diversity-solutions crowded in each point previously now spread over the flat minima region with the help of shaking operator.

According to the literature, the sharing implementation could change the result. ${ }^{14,17}$ To test the sharing approach the continuous problem was chosen (Figure 11a and 12a). The results of this test in terms of the runs contribution histogram and GHOST cross-section are shown in Figures 11c and 12c. It can be seen that the GHOST representation better resembles the original one, and also the runs contribution becomes more even. However, the distribution of $\chi^{2}$ is worse (see the minimum value and the distribution width in "sharing" column of Table 2). This result was not good
Table 2. Comparison of the $\chi^{2}$ Distributions and Solution Densities for the Different Multirun HEO-GHOST Approaches on the Synthetic 15-Component Spectrum that Simulates Quasicontinuous Distribution of Spectral Parameters ${ }^{a}$

\begin{tabular}{lcccc}
\hline criteria & 200 runs & 20 runs & sharing & shaking \\
\hline$\chi^{2} \min$ & 1.2 & 1.2 & 1.7 & 1.2 \\
$\sigma\left(\chi^{2}\right)$ & 0.9 & 0.4 & 1.3 & 0.9 \\
$\rho_{\max 0}$ & 69.5 & 75.7 & 69 & 66.1 \\
\multicolumn{5}{c}{ See also caption to Figure 12.} \\
\hline
\end{tabular}

enough, even when we increased the population size from 300 to 600 (to keep convergence at the same level due to the sharing implementation).

Grid Problem and Shaking. By careful analysis of the parameters in the resulting solution distribution, we found the origin of the unsuccessful implementation of the sharing approach - the shortcoming of the three-point crossover, one of the most important operators in the GA algorithm. "Genetic material" related to good model parameters spreads and copies among individuals in the population. After a few tens of generations the population forms a "grid" in the search space (Figure 9) as a consequence of the rough action of the 3-point crossover operator. This leads to the loss of solution diversity.

In the HEO algorithm only a local search operator is capable of restoring the diversity and eliminating the "grid", but due to the high computational cost and extremely high 
(a)

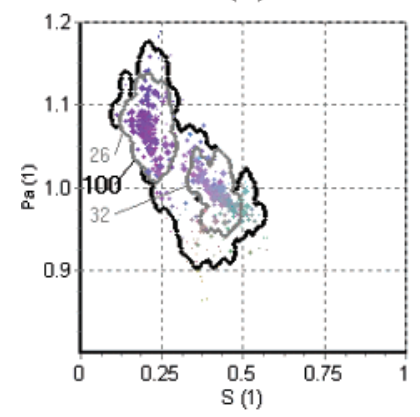

(b)

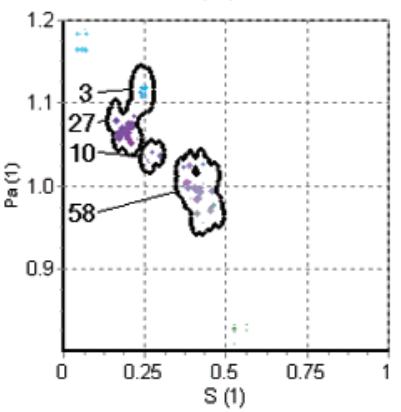

(c)

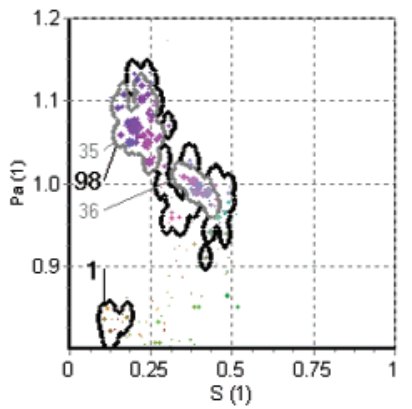

(d)

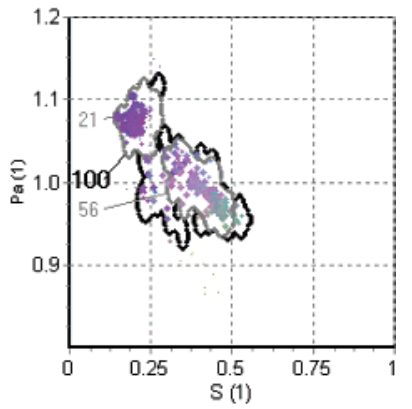

Figure 11. Comparison of the effectiveness of different multirun HEO-EnDashGHOST approaches on the synthetic 15-component spectrum that simulates a quasicontinuous distribution of spectral parameters. (a) GHOST as a result of 200 runs of original HEO routine; (b) GHOST as a result of 20 runs of the original HEO routine; (c) GHOST as a result of 20 runs of the modified HEO routine that includes sharing operator; and (d) GHOST as a result of 20 runs of the modified HEO routine that includes shaking operator as described in the text.
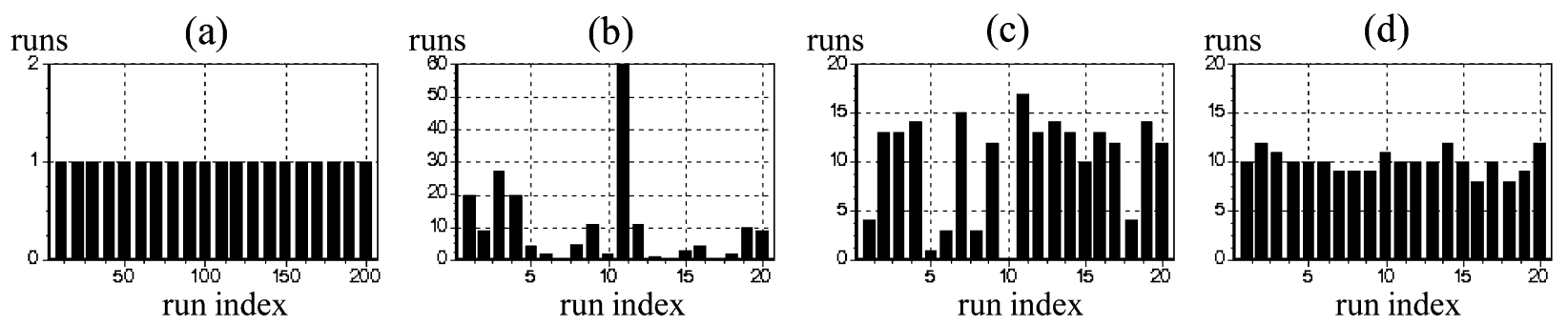

Figure 12. Comparison of runs contribution histograms of different multirun HEO - GHOST approaches on the synthetic 15-component spectrum that simulates a quasicontinuous distribution of spectral parameters. (a) GHOST as a result of 200 runs of original HEO routine; (b) GHOST as a result of 20 runs of the original HEO routine; (c) GHOST as a result of 20 runs of the modified HEO routine that includes sharing operator; and (d) GHOST as a result of 20 runs of the modified HEO routine that includes shaking operator as described in the text.

(a)
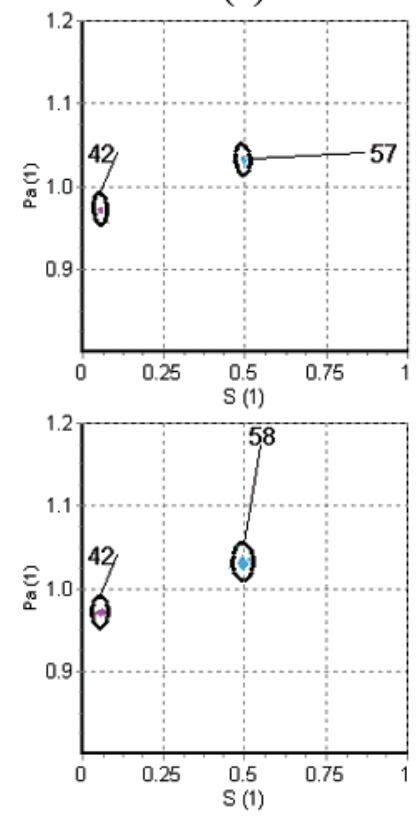

(b)
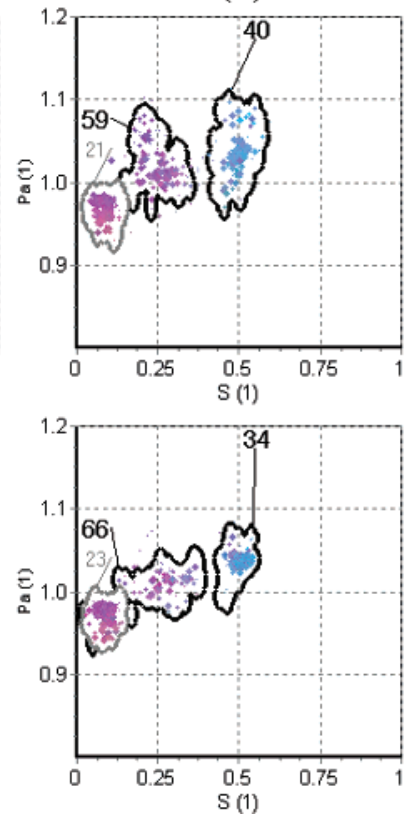

(c)
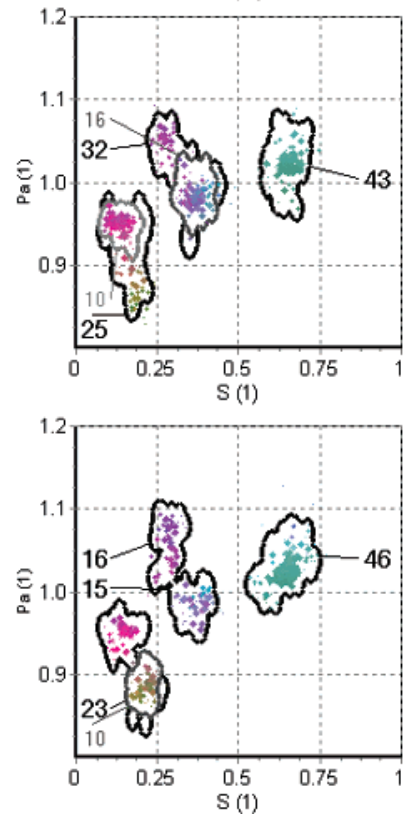

(d)
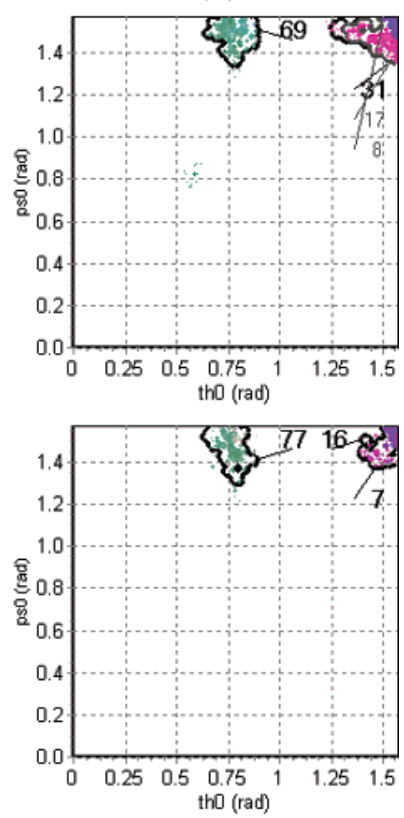

Figure 13. GHOSTs comparison of the original-HEO approach with 200 runs (above) versus modified-HEO (with shaking) based on 20 runs (bellow): (a) for synthetic discrete 2D spectrum that was constructed from two spectral components with the known parameter set and optimized as unknown one; (b) synthetic quasicontinues spectrum (see the caption to Figure 12); (c) spectrum of the real membranes of breast cancer cells MT1 in the exponential phase of growth: MT1 breast cancer cells were seeded at approximately $10^{6}$ cells in a culture flask with surface area of $75 \mathrm{~cm}^{2}$, spin labeled with the methyl ester of 5-doxyl palmitate, MeFASL(10,3), and measured under the same conditions as the membranes of horse neutrophils (see the caption to Figure 6); (d) spectrum of the spin labeled (maleimide spin label) cystein mutant of major coat protein of bacteriophage M13 at amino acid position 46 reconstituted in dimyristoylphosphatidylcholine lipid bilayer. ${ }^{31}$

impact on the convergence to local minima the probability of the Downhill-Simplex local search operator should be and is very low. Therefore the local search operator cannot be used to maintain population diversity. Instead, a new idea of "shaking" was introduced in our work keeping the standard crossover. As it was described in the Methods section, the shaking operator introduces a small deviation in parameters and thereby eliminates the effect of the "grid". 

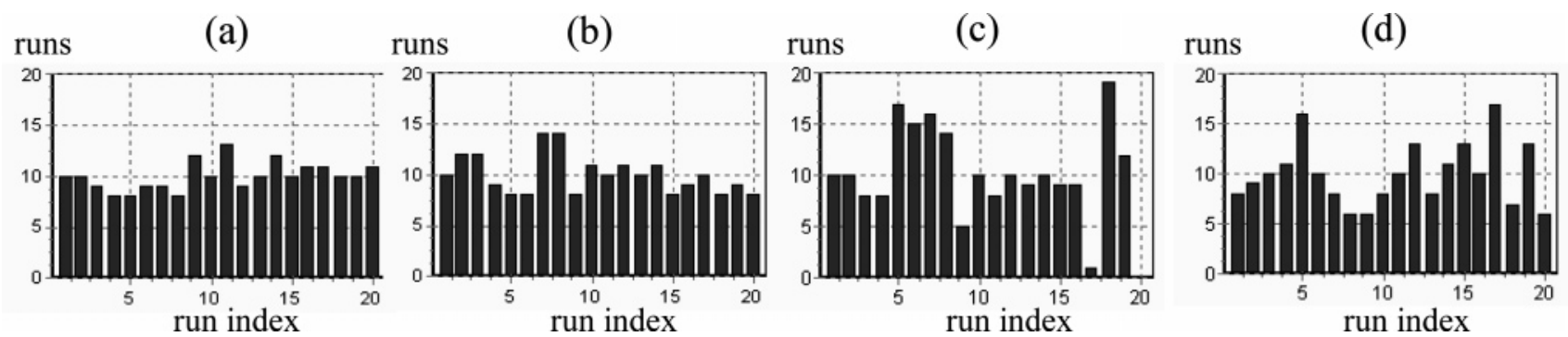

Figure 14. Runs contribution histogram for the modified HEO approach based on 20 runs with the implemented shaking operator. For the description of the samples (a)-(d) see the caption to Figure 13.

Indeed, the implementation of the shaking operator allowed the algorithm to overcome the solution crowding and increased the population diversity already in a single run. This result is shown in Figure 10 for a continuous problem that represents the most extreme case of the complexity.

The results of the implemented shaking algorithm are shown in Figures 11 and 12. One can see that the shaking operator considerably improves the result of a single run as the GHOST pattern from 20 runs (Figure 11d) is very similar to the original one (Figure 11a), the runs contribution histogram is very even (Figure 12d), and finally the distribution of $\chi^{2}$ is very good (Table 2). This approach therefore enables us to reduce the number of HEO runs while preserving the quality of the final result (Figures $11 \mathrm{~d}$ and $12 \mathrm{~d}$ and Table 2). Therefore by using this new algorithm, we are able to speed up the optimization process by a factor of $5-7$.

In further tests, the algorithm with the new shaking operator was also applied to several experimental and synthetic spectra in order to cover a wide range of possible systems related to discrete and continuous problems. The results of characterizations of four different examples are shown in Figure 13, where the GHOST diagrams of different approaches are compared (original "200-runs" approach is compared against "shaking-20-runs" approach). The GHOST diagrams are very similar, confirmed also by the comparison of the averaged values and the distribution widths of the condensed parameters (table is not shown).

\section{CONCLUSION}

To reduce the computational demand of the original multiple HEO approach, we developed and implemented a novel shaking operator and carried out an extensive testing on various spectra that represent a wide range of possible applications. With the modified optimization algorithm we succeeded in keeping the quality of the characterization, thereby considerably reducing the computational time of the EPR spectrum analysis by a factor of 5-7. With this successful modification the application of advanced EPR spectra analysis ${ }^{1}$ to complex biosystems, such as biological membranes and membrane proteins, becomes more feasible. Further numerical calculations on both synthetic and experimental data should prove the advantages of the implemented modifications and hopefully find new possibilities to improve and speed up EPR spectra analysis.

\section{ACKNOWLEDGMENT}

This work was carried out with the financial support of the Ministry of Higher Education, Science and Technology of the Republic of Slovenia through the Slovenian Research Agency. Initiation of the collaboration between the authors was enabled by the financial support from EC COST D22 Action.

\section{REFERENCES AND NOTES}

(1) Štrancar, J.; Koklič, T.; Arsov, Z.; Filipič, B.; Stopar, D.; Hemminga, M. A. Spin Label EPR-based Characterization of Biosystem Complexity. J. Chem. Inf. Model. 2005, 45, 394-406.

(2) Columbus, L.; Hubbell, W. L. A new spin on protein dynamics. Trends Biochem. Sci. 2002, 27, 288-295.

(3) Štrancar, J.; Koklič, T.; Arsov, Z. Soft picture of lateral heterogeneity in biomembranes. J. Membr. Biol. 2003, 196, 135-146.

(4) Budil, D. E.; Lee, S.; Saxena, S.; Freed, J. H. Nonlinear-Least-Squares Analysis of Slow-Motion EPR Spectra in One and Two Dimensions Using a Modified Levenberg-Marquardt Algorithm. J. Magn. Reson. A 1996, 120, 155-189.

(5) Robinson, B.; Thomann, H.; Beth, A.; Fayer, P.; Dalton, L. R. The phenomenon of magnetic resonance: Theoretical considerations. In EPR and Advanced EPR Studies of Biological Systems; Dalton, L. R., Ed.; CRC Press: Boca Raton, FL, 1985; pp 11-110.

(6) Schneider, D. J.; Freed, J. H. Calculating Slow Motional Magnetic Resonance Spectra: A User's Guide. In Biological Magnetic Resonance: Spin Labeling, Theory and Applications; Berliner, L. J., Reuben, J., Eds.; Plenum Press: New York, 1989; pp 1-76.

(7) Štrancar, J.; Šentjurc, M.; Schara, M. Fast and accurate characterization of biological membranes by EPR spectral simulations of nitroxides. J. Magn. Reson. 2000, 142, 254-265.

(8) Schindler, H.; Seelig, J. EPR spectra of spin labels in lipid bilayers. J. Chem. Phys. 1973, 59, 1841-1850.

(9) Marsh, D. Electron Spin Resonance: Spin Labels. In Membrane Spectroscopy; Grell, E., Ed.; Springer-Verlag: Berlin, 1981; pp 51142.

(10) Nordio, P. L. General magnetic resonance theory. In Spin Labeling, Theory and Application; Berliner, L. J., Ed.; Academic Press: New York, 1976; pp 5-51.

(11) Filipič, B.; Štrancar, J. Tuning EPR spectral parameters with a genetic algorithm. Appl. Soft Computing 2001, 1, 83-90.

(12) Goldberg, D. E. Genetic Algorithms in Search, Optimization and Machine Learning; Addison-Wesley: Reading, PA, 1989.

(13) Pétrowski, A. A. Clearing procedure as a niching method for genetic algorithms. In 3rd IEEE International Conference on Evol. Comput.; 1996; pp 798-803.

(14) Mahfoud, S. W. Niching Methods for Genetic Algorithms, Ph.D. Thesis, University of Illinois at Urbana-Champaign, Urbana, 1995.

(15) Beasley, D.; Bull, D. R.; Martin, R. R. A Sequential Niche Technique for Multimodal Function Optimization. Evol. Comput. 1993, 1, 101125 .

(16) Glover, F. Tabu Search - Part I. ORSA J. Comput. 1989, 1, 190206.

(17) Goldberg, D.; Richardson, J. Genetic algorithms with sharing for multimodal function optimization. In 2nd International Conference on Genetic Algorithms; Grefenstette, Ed., 1987; pp 41-49.

(18) De Jong, K. A. An Analysis of the Behavior of a Class of Genetic Adaptive Systems, Doctoral Dissertation, University of Michigan, 1975.

(19) Li, J. P.; Balazs, M. E.; Parks, G. T.; Clarkson, P. J. A species conserving genetic algorithm for multimodal function optimization. Evol. Comput. 2003, 11, 107-109.

(20) Spears, W. Simple Subpopulation Schemes. In 3rd Annual Conference on Evol. Programming; 1994; pp 296-307. 
(21) Deb, K. Genetic Algorithms in Multimodal Function Optimization, Master's Thesis, University of Alabama, 1989.

(22) Streichert, F.; Stein, G.; Ulmer, H.; Zell, A. A Clustering Based Niching Method for Evolutionary Algorithms. In Genetic Evol. Comput. Conf., Lect. Notes Comput. Sci. 2003, 2723, 644-645.

(23) Yin, X.; Germay, N. A Fast Genetic Algorithm with Sharing Scheme Using Cluster Analysis Methods in Multimodal Function Optimization. In Interernational Conference on Artificial Neural Nets and Genetic Algorithms; 1993; pp 450-457.

(24) Ursem, R. K. Multinational Evolutionary Algorithms. In Congress of Evol. Comput.; 1999; Vol. 3, pp 1633-1640.

(25) Gordon, V. S.; Whitley, D.; Bohn, A. Dataflow parallelism in genetic algorithms. In Parallel Problem Solving from Nature 2; Manner, R. Manderick, B., Eds.; Elsevier Science: Amsterdam, The Netherlands, 1992; pp 533-542.

(26) Bessaou, M.; Pétrowski, A.; Siarry, P. Island model cooperating with speciation for multimodal optimization. In 6th International Conference on Parallel Problem Solving From Nature; Schoenauer, M. et al., Eds.; Paris, France, 2000; pp 437-446.
(27) Martin, W. N.; Lienig J.; Cohoon, J. P. Island (migration) models: evolutionary algorithms based on punctuated equilibria. In Handbook of Evolutionary Computation; Back, T., Fogel, D. B., Michalewicz, Z., Eds.; Institute of Physics Publishing: Bristol, U.K., 1997; pp C6.3 1-C6.3:1.

(28) Darwen, P. J.; Xin, Y.. Every Niching Method has its Niche: Fitness Sharing and Implicit Sharing Compared. In 4th Int. Conf. Parallel Problem Solving from Nature, Lect. Notes Comput. Sci. 1996, 1141, 398-407.

(29) Mahfoud, S. W. Simple Analytical Models of Genetic Algorithms for Multimodal Function Optimization; Technical Report IlliGAL Report No 93001; 1993.

(30) Sareni, B.; Krähenbühl, L. Fitness Sharing and Niching Methods Revisited. In IEEE Trans. Evol. Comput.; 1998; Vol. 2.

(31) Stopar, D.; Spruijt, R. B.; Wolfs, C. J. A. M.; Hemminga, M. A. Protein-lipid interactions of bacteriophage M13 major coat protein. Biochim. Biophys. Acta 2003, 1611, 5-15.

CI0501589 